\title{
Resorption of impacted teeth as a marker of squamous cell carcinoma infiltrating the mandible*
}

\author{
Sathesh Balasundram ${ }^{1 \#}$, Nicolas Dulguerov ${ }^{2}$, Donald Murray Walker ${ }^{3}$, Colin Liew ${ }^{2}$ \\ ${ }^{1}$ Eastman Dental Institute, University College London, London, UK \\ ${ }^{2}$ Department of Oral and Maxillofacial Surgery, University College London Hospital, London, UK \\ ${ }^{3}$ University College London Hospital, London, UK \\ Email: ${ }^{\text {satbala@yahoo.com }}$
}

Received 21 January 2013; revised 24 February 2013; accepted 4 March 2013

\begin{abstract}
The invasion of the mandible by oral squamous cell carcinoma has been firmly demonstrated by anatomical and histological studies. Although squamous cell carcinomas may induce resorption of the roots of standing teeth, there have been no reports of them also causing resorption of the crowns of teeth. A 73year-old patient presented with an invading buccal squamous cell carcinoma. Radiological investigations showed tumour invasion of the dentate region of the left mandible and resorption of the crowns of right and left mandibular impacted third molars. The patient underwent a left segmental mandibulectomy and histopathological examination of the resected specimen revealed resorption of the enamel and dentine of an unerupted impacted third molar closely associated with the infiltrating tumour. Thus infiltrating carcinomas may rarely cause resorption of the crowns as well as the roots of unerupted teeth and can be a valuable marker of the extent of spread of a tumour for the planning of complete surgical resection. These dental changes must be distinguished radiologically from idiopathic changes in unerupted teeth in the absence of malignancy.
\end{abstract}

Keywords: Squamous Cell Carcinoma; Invasion; Tooth Resorption

\section{INTRODUCTION}

Advanced oral cancer not infrequently invades the bone of the mandible. Lesions adjacent to the mandible often involve the bone by direct extension and less commonly by other routes. The prevalence of mandibular bone involvement ranges from $12 \%$ to $56 \%[1,2]$.

The prognostic impact of mandibular invasion by oral

${ }^{*}$ Conflict of interest: none declared. Financial disclosure: none.

${ }^{\#}$ Corresponding author. squamous cell carcinoma is controversial. Ogura et al. (2002) showed that bony invasion identified on dental computed tomography scan (CT) images was a significant prognostic factor in cervical metastases [3]. Survival was significantly influenced by positive soft tissue margins but not bone invasion or the type of resection [4]. Patel et al. (2008) [5] assessed the effect that the extent of bone invasion has on recurrence and survival in patients treated with marginal and segmental mandible resection. There was no correlation with presence or extent of bone invasion. Survival at 5 years was $71 \%$ and this correlated with bone invasion and involved margins, but not with extent of mandible invasion or resection. Pandey et al. (2009) [6] found no significant difference in survival between patients with pathologically positive mandible with those not having mandibular involvement.

However, Ebrahimi et al. (2011), [7] demonstrated that tumor size and medullary bone invasion are independent predictors of reduced survival. In contrast, tumors with bone invasion limited to the cortex had a similar prognosis to those without bone invasion.

Where a tumour is close to the maxilla or mandible adequate clinical and radiographic assessment is essential to determine the possibility of bone invasion. The clinical presentation of oral squamous cell carcinoma includes hard, fixed ulcers with raised margins, non healing white or red patches, fleshy polyps, punched-out ulcers, indurated plaques or tethering mucosa. A panoramic radiograph of the mandible is frequently used for the imaging, but a more detailed examination is available with CT, magnetic resonance imaging (MRI), bone scan and single photon emission computed tomography (SPECT) scan.

It is generally recognized that there are two types of invasion of the mandible by oral squamous cell carcinoma. With the invasive or expansive pattern of disease, fingers and islands of tumour advance independently into the cancellous spaces, [8,9] associated with increased numbers of osteoclasts. Cytokines such as IL-1 or PTHrP 
released by tumour or stromal cells can induce RANKL synthesis by stromal fibroblasts or osteoblasts. In turn, RANKL can activate osteoclast formation [10]. Subperiosteal new bone formation and osteogenesis are increased. In the "erosive" pattern, the tumour advances on a broad front with a well-defined connective-tissue layer and active osteoclasts separating the tumour from the bone [8]. Destruction of bone by tumour is caused by osteoclasts rather than by tumour cells directly [11].

The common causes of resorption of unerupted teeth include cystic changes involving the reduced enamel epithelium, trauma induced resorption and inflammatory resorption. Idiopathic resorption of unerupted teeth in the absence of other disease is not uncommon, especially in long standing impacted teeth. This is important when loss of tooth structure is observed adjacent to an invading carcinoma. Although there have been case reports of root resorption due to squamous cell carcinoma masquerading as periapical infections [12], there have been few if any systematic studies of the frequency or distribution of these changes in dentate jaws associated with.

The following report describes a rare case of a patient with resorption of the crown and root of an impacted tooth associated with a buccal squamous cell carcinoma. The distinction between this and idiopathic external resorption is discussed. The significance of this finding in planning complete surgical resection is emphasized.

\section{CASE REPORT}

A 73-year-old Caucasian lady presented with a gingival swelling over the left molar region of the mandible which was first noticed 4 months earlier with gradual increase in size and occasional bleeding. There was no history of toothache or pain. She had had two courses of antibiotics with no improvement.

Her medical history was not significant.

There was an endophytic, ulcerated, slough-like mass approximately of $2 \times 3 \mathrm{~cm}$ in the left buccal sulcus adjacent to the lower left first and second molar. There was no paresthesia of the inferior alveolar nerve of the mandible.

Panoramic radiograph (Figure 1(a)) did not reveal bony changes but both lower third molars were unerupted and their crowns partially resorbed.

On computed tomography images (Figure 1(b)) an illdefined bony erosion of the buccal aspect of the superior lateral cortex of the mandible around the left lower five and six was noticed. There was lucency around the root and crown of an impacted left lower eight.

An incisonal biopsy of the left buccal sulcus swelling confirmed the diagnosis of squamous cell carcinoma.

The patient underwent a left segmental mandibulectomy and a left selective neck dissection with reconstruction using a free deep circumflex iliac artery flap (iliac

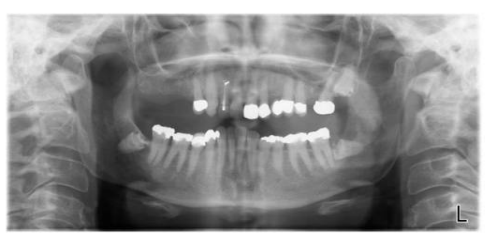

(a)

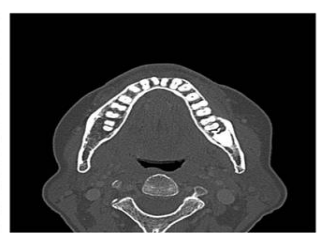

(b)
Figure 1. (a) Orthopantomograph featuring resorption of the crowns of both unerupted mandibular third molars; (b) Computed Tomography image showing bone erosion of the buccal aspect of the superior lateral cortex of the mandible around the left lower five and six and lucency around the root and crown of an impacted left lower eight.

crest free flap).

The histopathologic examination of the surgical specimen revealed tumour invasion buccal to the lower second molar almost to the root apex and abutting the horizontally lying unerupted third molar (Figure 2). There was evidence of bone resorption (Figure 3(a)) along with resorption of dentine and enamel of the impacted third molar (Figure 3(b)). Osteoclasts were noted on the surface of the enamel near the advancing front of the tumour (Figures 4(a) and (b)). There was no nodal metastasis.

The patient received radiotherapy postoperatively. The patient has been followed-up for 4 years. She remains disease free.

\section{DISCUSSION}

Tumours that approach or invade the mandible require specific understanding of the mechanism of bone involvement and invasion. This ensures the appropriate approaches and management of the mandible.

In early invasion, clinical examination has been found to be sensitive, however the best results are obtained by combining a good clinical examination, intra oral periosteal stripping and supplemented with one or more radiologic investigations [13]. Panorex radiographs play an important role in the detection of bone invasion by oral carcinoma especially in determining the superior-inferior extent of the tumour in the bone, which is one of the most important factors influencing the selection between rim and segmental resection of the mandible. Rao et al. (2004) [2] showed that the orthopantomographs (OPG) had a sensitivity of $92 \%$, failing to detect mandibular bone invasion in $8 \%$ cases. The false positive rate for OPG in the study was $12 \%$. These observations agree with those reported in the literature [2,14-16]. Routine radiographs including OPG are unable to detect initial bone invasion until $30 \%$ of the mineral has lost, accounting for the false negative reports. On the other hand, CT, MRI, and bone scintigraphy have been found to be more useful with sensitivity and specificity ranging from $85 \%$ to $95 \%$. 


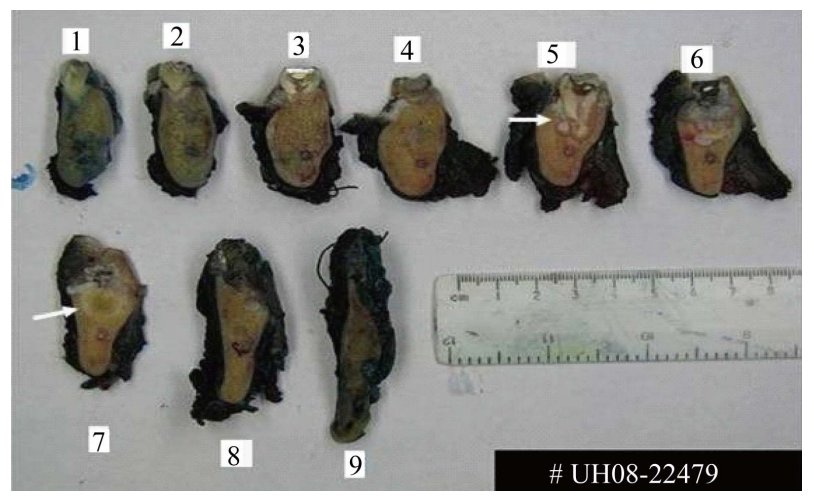

Figure 2. Coronal plane slices of the resection specimen of the left mandible, molar region anterior to the ascending ramus. The tumour has infiltrated the buccal surface of the second molar almost to the root apex (arrows) and abuts the crown of the horizontally lying, third molar (arrows).

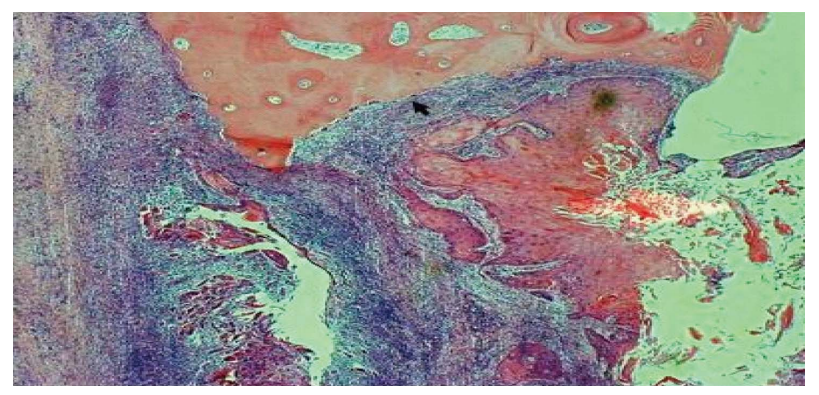

(a)

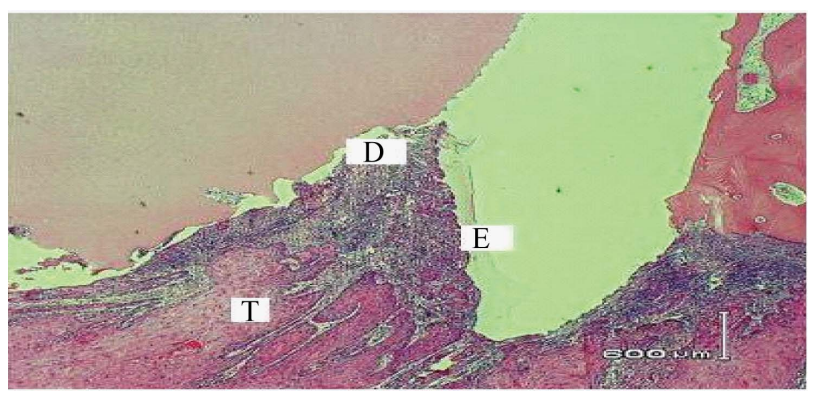

(b)

Figure 3. (a) Resorption of mandible by tumour; (b) Tumour inducing resorption of dentine and enamel of third molar.

When a tumour involves bone, both osteoclastic and osteoblastic activity can be stimulated [17]. Once the residual alveolar bone of the jaws has been resorbed during the erosive phase of tumour spread, involvement of the deeper basal bone tends to be characterized by the invasive pattern of spread [8].

O’Reilly et al. (2000) [18] noted such aggressive behaviour may show a predilection for causing tooth movement and displacement or resorption of roots. This is consistent with the finding in this case with the tooth abutting the tumour affected, and with resorption of the crown and erosion of pericoronal and periapical bone. The osteoclastic enamel and dentine resorption of the

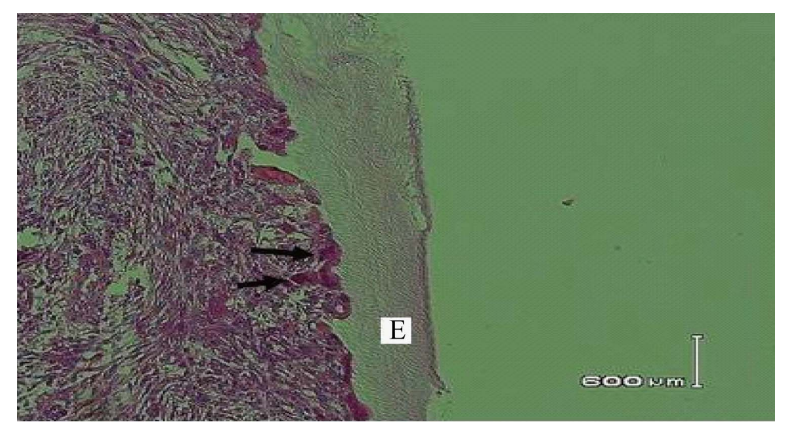

(a)

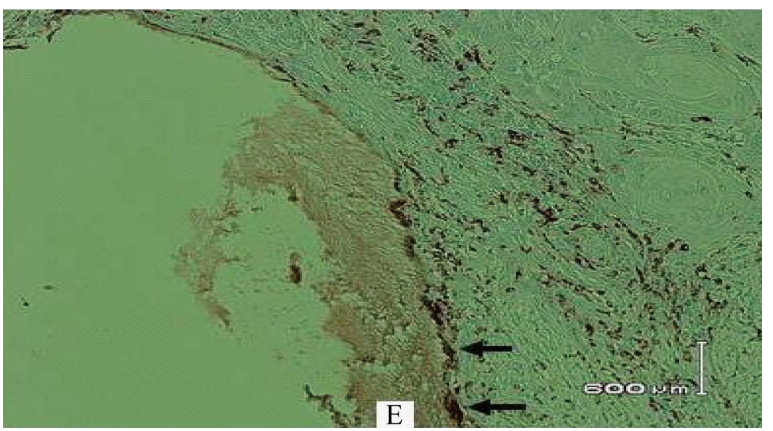

(b)

Figure 4. (a) Osteoclasts (arrows) on surface of enamel; (b) Osteoclasts (arrows) stained by immunohistochemistry for CD68, on surface of enamel (E).

unerupted molar is the unusual feature in our case. The appearances in the OPG might at first inspection be misinterpreted as bilateral idiopathic resorption of the crowns of the unerupted third molars. In fact, the histology showed that the resorption of the left mandibular third molar was due to involvement by the carcinoma, which is of significance when planning complete resection. By contrast, teeth undergoing idiopathic external resorption commonly also have histological evidence of focal repair by cementum or bone with reversal lines and sometimes focal ankylosis to adjacent bone.

We report that crown and root resorption of unerupted teeth may be a feature of invasion of the dentate mandible by squamous cell carcinoma. This would call for a meticulous radiological assessment of impacted teeth within the vicinity of the tumour. Resorption of unerupted teeth associated with malignancy has to be distinguished from idiopathic external resorption which as in our case can be quite similar radiographically and the final diagnosis may have to await histological assessment. Misdiagnosis of tooth resorption and the insensitivity of radiological staging could contribute to underestimation of the tumour extent and inadequate resection of the mandible.

\section{REFERENCES}

[1] Pandey, M., Rao, L.P., Das, S.R., Mathews, A., Chacko, 
E.M. and Naik, B.R. (2007) Patterns of mandibular invasion in oral squamous cell carcinoma of the mandibular region. World Journal of Surgical Oncology, 30, 12.

[2] Rao, L.P., Das, S.R., Mathews, A., Naik, B.R., Chacko, E. and Pandey, M. (2004) Mandibular invasion in oral squamous cell carcinoma: Investigation by clinical examination and orthopantomogram. International Journal of Oral and Maxillofacial Surgery, 33, 454-457. doi:10.1016/j.ijom.2003.10.006

[3] Ogura, I., Kurabayashi, T., Amagasa, T., Okada, N. and Sasaki, T. (2002) Mandibular bone invasion by gingival carcinoma on dental CT images as an indicator of cervical lymph node metastasis. Maxillofacial Radiology, 31, 339-343.

[4] O’Brien, C.J., Adams, J.R., McNeil, E.B., Taylor, P., Laniewski, P., Clifford, A. and Parker, G.D. (2003) Influence of bone invasion and extent of mandibular resection on local control of cancers of the oral cavity and oropharynx. International Journal of Oral and Maxillofacial Surgery, 32, 492-497.

[5] Patel, R.S., Dirven, R., Clark, J.R., Swinson, B.D., Gao, K. and O’Brien, C.J. (2008) The prognostic impact of extent of bone invasion and extent of bone resection in oral carcinoma. Laryngoscope, 118, 780-785.

[6] Pandey, M., Rao, L.P., Das, S.R. and Shukla, M. (2009) Tumor stage and resection margins not the mandibular invasion determines the survival in patients with cancers of oro-mandibular region. European Journal of Surgical Oncology, 35, 1337-1342.

[7] Ebrahimi, A., Murali, R., Gao, K., Elliott, M.S. and Clark, J.R. (2011) The prognostic and staging implications of bone invasion in oral squamous cell carcinoma. Cancer, 117, 4460-4467. doi:10.1002/cncr.26032

[8] Brown, J.S. and Browne, R.M. (1995) Factors influencing the patterns of invasion of the mandible by oral squamous cell carcinoma. International Journal of Oral and Maxillofacial Surgery, 24, 417-426. doi:10.1016/S0901-5027(05)80471-0

[9] Chen, Y.L., Kuo, S.W., Fang, K.H. and Hao, S.P. (2011) Prognostic impact of marginal mandibulectomy in the presence of superficial bone invasion and the nononcologic outcome. Head \& Neck, 33, 708-713. doi:10.1002/hed.21530

[10] Kayamori, K., Sakamoto, K., Nakashima, T., Takayanagi, H., Morita, K., Omura, K., Nguyen, S.T., Miki, Y., Iimura, T., Himeno, A., Akashi, T., Yamada-Okabe, H., Ogata,
E. and Yamaguchi, A. (2010) Role of interleukin-6, and parathormone-related peptide in osteoclast formation associated with oral cancer: Significance of interleukin-6 synthesised by stromal cells in response to cancer cells. American Journal of Pathology, 176, 968-980. doi:10.2353/ajpath.2010.090299

[11] Van Cann, E.M., Slootweg, P.J., de Wilde, P.C., OtteHöller, I., Koole, R., Stoelinga, P.J. and Merkx, M.A. (2009) The prediction of mandibular invasion by squamous cell carcinomas with the expression of osteoclastrelated cytokines in biopsy specimens. International Journal of Oral and Maxillofacial Surgery, 38, 279-284. doi:10.1016/j.ijom.2009.01.005

[12] Hutchison, I.L., Hopper, C. and Coonar, H.S. (1990) Neoplasia masquerading as periapical infection. British Dental Journal, 168, 288-294. doi:10.1038/sj.bdj.4807171

[13] Pandey, M., Rao, L.P. and Das, S.R. (2009) Predictors of mandibular involvement in cancers of the oromandibular region. Journal of Oral and Maxillofacial Surgery, 67, 1069-1073. doi:10.1016/j.joms.2008.06.059

[14] Acton, C.H., Layt, C., Gwynne, R., Cooke, R. and Seaton, D. (2000) Investigative modalities of mandibular invasion by squamous cell carcinoma. Laryngoscope, 110, 20502055. doi:10.1097/00005537-200012000-00014

[15] Kalavrezos, N.D., Grätz, K.W., Sailer, H.F. and Stahel, W.A. (1996) Correlation of imaging and clinical features in the assessment of mandibular invasion of oral carcinomas. International Journal of Oral and Maxillofacial Surgery, 25, 439-445. doi:10.1016/S0901-5027(96)80079-8

[16] Söderholm, A.L., Lindqvist, C., Hietanen, J. and Lukinmaa, P.L. (1990) Bone scanning for evaluating mandibular bone extension of oral squamous cell carcinoma. Journal of Oral and Maxillofacial Surgery, 48, 252-257. doi:10.1016/0278-2391(90)90389-J

[17] McGregor, A.D. and MacDonald, D.G. (1988) Reactive changes in the mandible in the presence of squamous cell carcinoma. Head \& Neck Surgery, 10, 378-386. doi:10.1002/hed.2890100604

[18] O’Reilly, M., O’Reilly, P., Todd, C.E., Altman, K. and Schafler, K. (2000) An assessment of the aggressive potential of radiolucencies related to the mandibular molar teeth. Clinical Radiology, 55, 292-295. doi:10.1053/crad.1999.0346 